\title{
Development of superconducting crossbar- $H$-mode cavities for proton and ion accelerators
}

\author{
F. Dziuba, ${ }^{1}$ M. Busch, ${ }^{1}$ M. Amberg, ${ }^{1}$ H. Podlech, ${ }^{1}$ C. Zhang, ${ }^{1}$ H. Klein, ${ }^{1}$ W. Barth,${ }^{2}$ and U. Ratzinger ${ }^{1}$ \\ ${ }^{1}$ Institute for Applied Physics (IAP), University of Frankfurt, 60438 Frankfurt, Germany \\ ${ }^{2}$ GSI Darmstadt, Planckstraße 1, 64291 Darmstadt, Germany
}

(Received 30 November 2009; published 26 April 2010)

\begin{abstract}
The crossbar- $H$-mode $(\mathrm{CH})$ structure is the first superconducting multicell drift tube cavity for the low and medium energy range operated in the $H_{21}$ mode. Because of the large energy gain per cavity, which leads to high real estate gradients, it is an excellent candidate for the efficient acceleration in high power proton and ion accelerators with fixed velocity profile. A prototype cavity has been developed and tested successfully with a gradient of $7 \mathrm{MV} / \mathrm{m}$. A few new superconducting $\mathrm{CH}$ cavities with improved geometries for different high power applications are under development at present. One cavity $(f=$ $325 \mathrm{MHz}, \beta=0.16$, seven cells) is currently under construction and studied with respect to a possible upgrade option for the GSI UNILAC. Another cavity ( $f=217 \mathrm{MHz}, \beta=0.059,15$ cells) is designed for a cw operated energy variable heavy ion linac application. Furthermore, the EUROTRANS project (European research program for the transmutation of high level nuclear waste in an accelerator driven system, $600 \mathrm{MeV}$ protons, $352 \mathrm{MHz}$ ) is one of many possible applications for this kind of superconducting rf cavity. In this context a layout of the $17 \mathrm{MeV}$ EUROTRANS injector containing four superconducting $\mathrm{CH}$ cavities was proposed by the Institute for Applied Physics (IAP) Frankfurt. The status of the cavity development related to the EUROTRANS injector is presented.
\end{abstract}

DOI: 10.1103/PhysRevSTAB.13.041302

PACS numbers: 29.20.Ej, 28.65.+a

\section{SUPERCONDUCTING CH CAVITIES IN HIGH POWER PROTON AND ION ACCELERATORS}

International projects with high requirements regarding beam power and quality ask for new linac developments. In this context the superconducting crossbar- $H$-mode $(\mathrm{CH})$ cavity has been investigated for several years at the Institute for Applied Physics (IAP) of Frankfurt University [1]. This kind of cavity is particularly suitable for international high power applications with intense beam currents like the EUROTRANS [2] project because its well designed multicell geometry reduces the number of drift spaces between the cavities significantly compared to conventional low- $\beta$ ion linacs [3]. Furthermore, the superconducting $\mathrm{CH}$ structure delivers a better efficiency at high duty cycles in comparison with room temperature cavities. Most of the $\mathrm{CH}$ as well as interdigital- $H$-mode (IH) cavities are operated with the special KONUS (combined zero degree structure) beam dynamics, which decreases transverse rf defocusing effects and enables long lens free acceleration sections $[1,4,5]$. Compared to conventional beam dynamics methods, in KONUS dynamics the acceleration, the longitudinal as well as the transverse focusing, are separated. A typical KONUS period consists of three sections: (i) main accelerating section with synchronous phase $\varphi_{s}=0^{\circ}$; (ii) transversal focusing element (quadrupole triplet or solenoid); (iii) rebuncher section with $\varphi_{s} \approx$ $-35^{\circ}$ for longitudinal focusing. Additionally, $\mathrm{CH}$ cavities can also be operated with the equidistant multigap structure (EQUUS) beam dynamics concept [6]. All these mentioned components lead to high real estate gradients and to compact linear accelerators.
A 19-cell, $360 \mathrm{MHz}$ superconducting prototype $\mathrm{CH}$ cavity has been developed and tested at the IAP [7]. Gradients of $7 \mathrm{MV} / \mathrm{m}$, corresponding to an effective voltage gain of 5.6 MV, were reached [8]. In the framework of the high intensity pulsed proton injectors (HIPPI) research program, a tuning system pushing the cavity end cells has been developed [9]. After several vertical measurements, the cavity has been prepared for tests in a horizontal cryomodule (see Fig. 1). In the first step, a frequency shift of $400 \mathrm{kHz}$ was achieved with the slow tuner as expected [10]. In the next step the fast tuner system was tested. The tuning device, based on three piezo elements mounted in a socket ring, pushes one cavity end cell while the other end

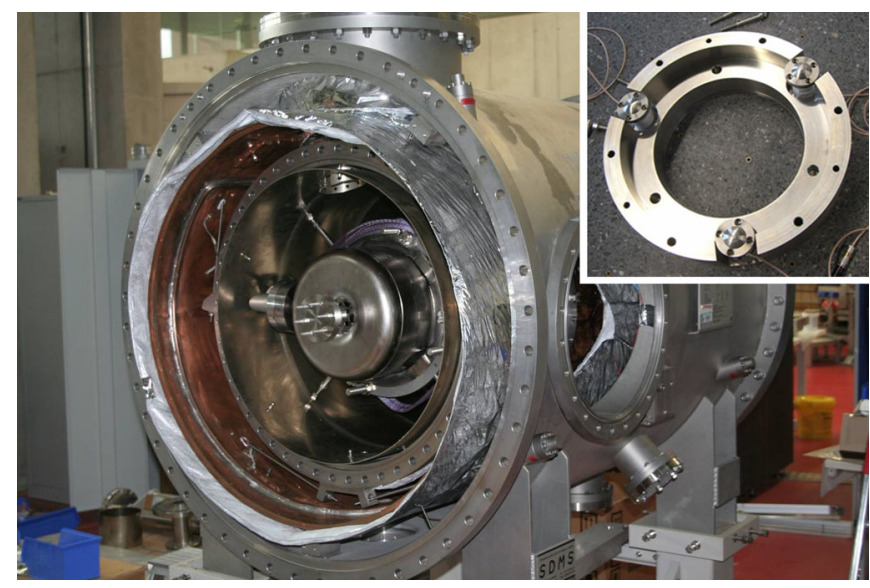

FIG. 1. (Color) $360 \mathrm{MHz}$ prototype $\mathrm{CH}$ cavity implemented in the horizontal cryomodule with a picture of the piezo tuning device. 


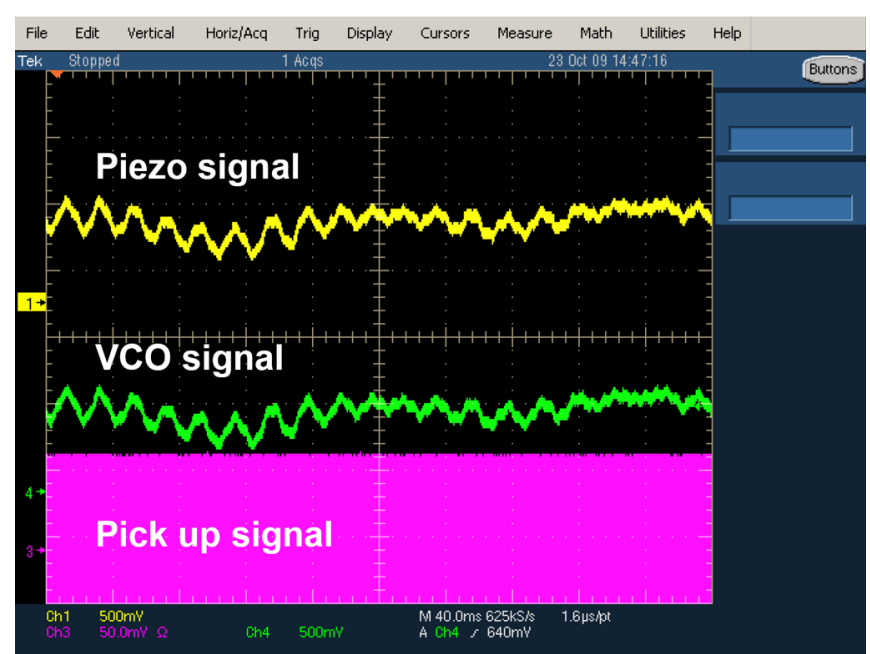

FIG. 2. (Color) Test of the piezo tuning system compensating frequency changes at $4 \mathrm{~K}$.

is fixed. This kind of tuning system leads to a change in capacitance and finally to a frequency shift. It is supposed to act against microphonics, Lorentz force detuning and fast pressure variations during operation. After the cavity was cooled down to $4 \mathrm{~K}$, a phase difference between generator and cavity signal caused by microphonics could be measured with a phase detector. This phase difference $\Delta \phi$ is directly proportional to a frequency deviation $\Delta f$. Finally, the voltage of a voltage controlled oscillator $\left(V_{\mathrm{VCO}}\right)$, which is proportional to $\Delta f$ and $\Delta \phi$ as well, was used to drive the piezo tuning device. Depending on this voltage the piezos change their length and deform the cavity. This results in a compensation of the frequency deviation shown by the constant field level of the pickup signal (see Fig. 2).

Encouraged by these promising results, a new prototype designed for high power applications was proposed (see Fig. 3). This new cavity, operated at $325 \mathrm{MHz}$ with a design beta of 0.16 , consists of seven cells and is presently under construction at Research Instruments (RI) GmbH, Bergisch Gladbach, Germany. It will be equipped with all necessary auxiliaries like power couplers, tuning systems, and helium vessel. The frequency is the third harmonic of the UNILAC at GSI. To demonstrate the cavity capabilities under realistic accelerator environments, it will be tested in a horizontal cryomodule together with two superconducting solenoids. In addition, it is planned to test the cavity in 2012 at full rf power with a $10 \mathrm{~mA}, 11.4 \mathrm{AMeV}$ uranium beam delivered by the UNILAC. In Table I the main parameters of the new cavity layout are summarized.

To fulfill the requirements for high power operations, the geometry of the new $\mathrm{CH}$ cavity has been optimized. The main changes compared to the first $\mathrm{CH}$ prototype are: (i) optimized stem geometry; (ii) inclined end stems; (iii) additional flanges at the tank caps for cleaning procedures; (iv) two bellow tuners inside the cavity; and (v) port for large power coupler due to beam operation.

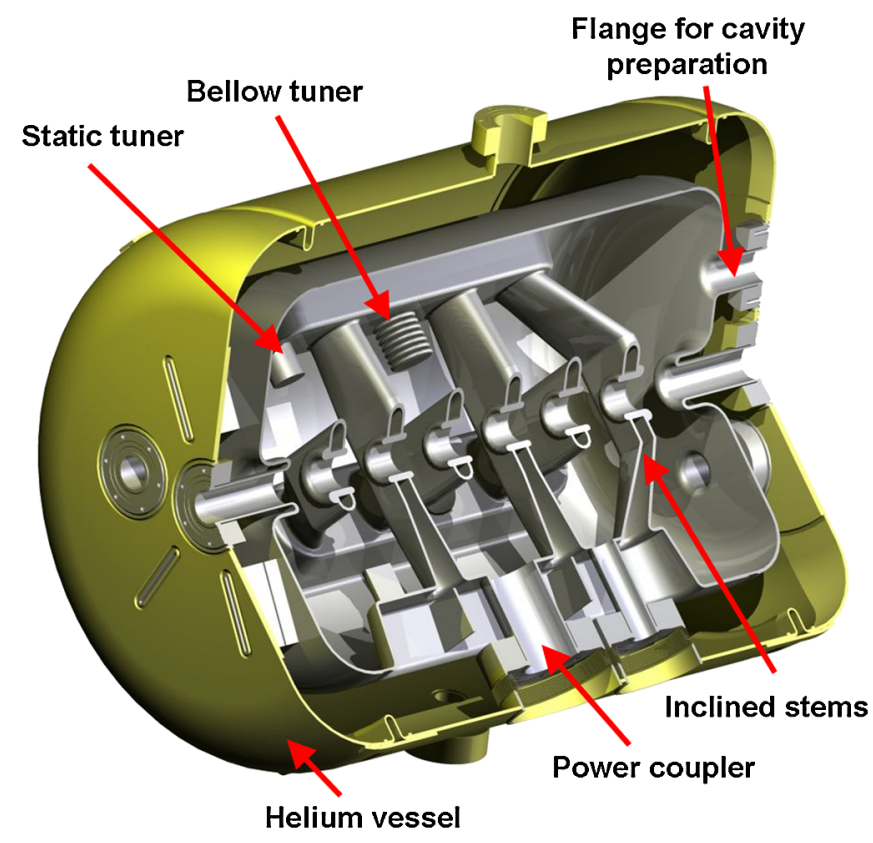

FIG. 3. (Color) Design of the superconducting $325 \mathrm{MHz} \mathrm{CH}$ cavity.

The stem geometry has been optimized, respectively, to the peak fields and to accommodate a sufficiently large power coupler into the girders. Inclined stems at the end cells lead to a very homogeneous field distribution along the beam axis because their new orientation increases the inductance as well as the electric field in this area [11]. A comparison of the electric field distribution for straight and inclined end stems is shown in Fig. 4. This kind of end cell variation reduces the longitudinal dimensions of the cavity by about $20 \%-25 \%$, as an extended end drift tube is not needed in that case for field flattening. Using inclined end stems has also great advantages in terms of the beam dynamics of high intensity linacs: A reduction of the drift tube lengths between neighbored drift tube sections is causing a reduced growth of the bunch length and an increased longitudinal phase space acceptance of the linac. For a comparison, the first $360 \mathrm{MHz}$ prototype $\mathrm{CH}$ cavity

TABLE I. Main parameters of the $325 \mathrm{MHz} \mathrm{CH}$ cavity.

\begin{tabular}{lcc}
\hline \hline Material & & Bulk niobium \\
$\beta$ & & 0.16 \\
Frequency & $\mathrm{MHz}$ & 325.224 \\
Total length & $\mathrm{mm}$ & 505 \\
Cavity diameter & $\mathrm{mm}$ & 352.6 \\
Accelerating cells & & 7 \\
$E_{a}(\beta \lambda$ definition $)$ & $\mathrm{MV} / \mathrm{m}$ & 5 \\
$E_{p} / E_{a}$ & & 5.1 \\
$B_{p} / E_{a}$ & $\mathrm{mT} /(\mathrm{MV} / \mathrm{m})$ & 13 \\
$G$ & $\Omega$ & 64 \\
$R_{a} / Q_{0}$ & $\Omega$ & 1248 \\
$R_{a} R_{s}$ & $\mathrm{k} \Omega^{2}$ & 80 \\
\hline \hline
\end{tabular}




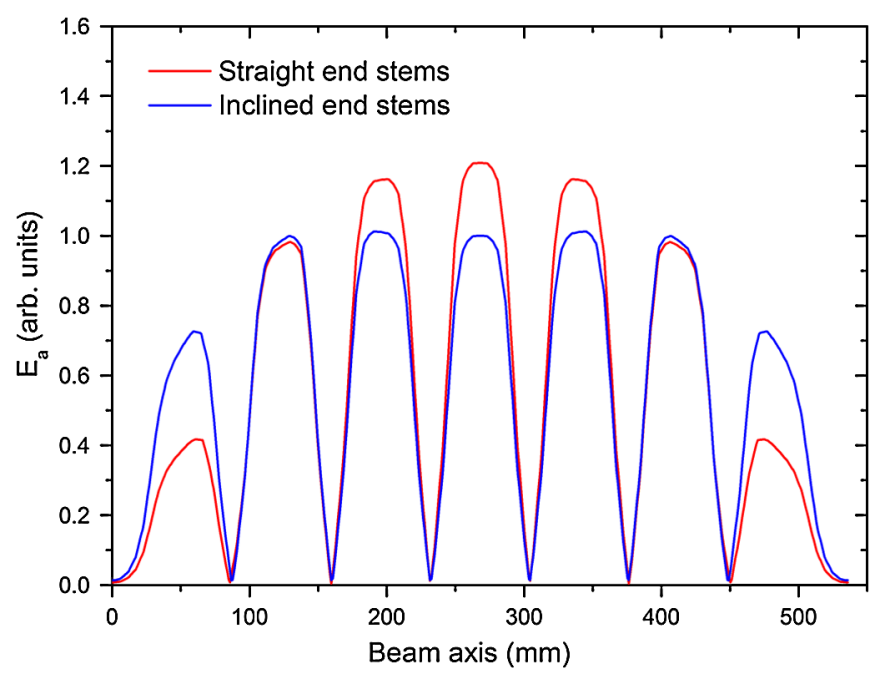

FIG. 4. (Color) Simulation of the electric field distribution for different end stem geometries.

had half drift tube lengths of $131 \mathrm{~mm}$, while in the actual $325 \mathrm{MHz} \mathrm{CH}$-cavity design, with inclined end stems, this length was shortened to $46 \mathrm{~mm}$.

The disadvantage of inclined end stems is an increased magnetic peak field. Experiences with the first superconducting prototype cavity showed that an intensive surface preparation is essential for the performance of the cavity. Therefore, the flanges at the tank caps provide a promising way to process the cavity surface with buffered chemical polishing and high pressure rinsing.

The cavity tuning will be done by capacitive tuners welded into the girders between the stems to reach the design frequency, which will be controlled during the fabrication and adapted accordingly. For that reason the $325 \mathrm{MHz} \mathrm{CH}$ cavity will contain four static tuners with cylindrical geometry. Their height will be fixed after the frequency adjustment. To calculate their influence inside the cavity, rf simulations have been performed. Figure 5

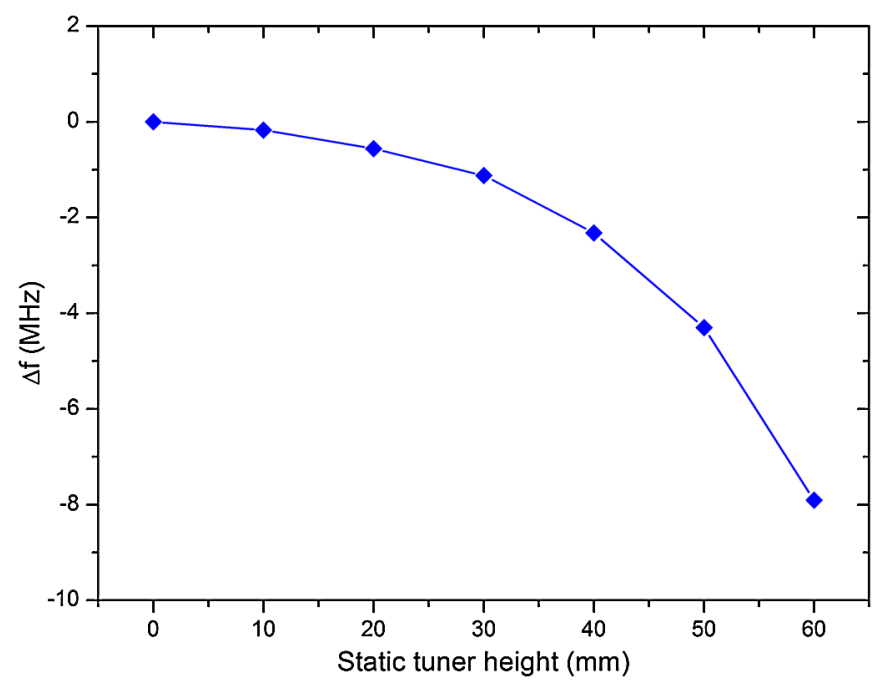

FIG. 5. (Color) Simulated tuning range of the four static tuners.
TABLE II. Frequency shift and total range of the fast and slow bellow tuner.

\begin{tabular}{lcc}
\hline \hline Parameter & Slow tuner & Fast tuner \\
\hline No. & 1 & 1 \\
Frequency shift & $150 \mathrm{kHz} / \mathrm{mm}$ & $150 \mathrm{~Hz} / \mu \mathrm{m}$ \\
Total range & $\pm 150 \mathrm{kHz}( \pm 1 \mathrm{~mm})$ & $\pm 750 \mathrm{~Hz}( \pm 5 \mu \mathrm{m})$ \\
\hline \hline
\end{tabular}

shows the simulated frequency range depending on the tuner height. This tuning concept has been proven already during the fabrication of the superconducting $360 \mathrm{MHz}$ prototype cavity [7]. Additionally, two dynamic bellow tuners are provided reaching a tuning range of several hundred kHz. One tuner will be driven by a piezo element to compensate limitations like microphonics, Lorentz force detuning, etc., during beam operation. The other one, driven by a step motor, will act as a slow tuner to readjust frequency changes and pressure effects at $4.2 \mathrm{~K}$. By using these bellow tuners, the required longitudinal space for cavity installation will be minimized in contrast to a tuner system pushing on the end cells. In order to estimate the frequency gain $\Delta f / \Delta h$ of the dynamic tuners, the height of the static ones was kept constant while increasing the bellow tuners continuously. Table II shows that at a point of approximately $53 \mathrm{~mm}$ tuner height a shift of $150 \mathrm{kHz} / \mathrm{mm}$ is achievable for both bellow tuners, which is sufficient for tuning during beam operation.

To analyze their mechanical behavior, several static structural simulations have been carried out where a bellow tuner model was exposed to a range of static forces. The three-dimensional contour plot of Fig. 6 illustrates the deformation of the seven segmented tuner after pushing on the central stem with a force of up to $450 \mathrm{~N}$ (top) and the deformation of the tuner depending on the lateral force (bottom). The maximum effect appears at the segment on top of the tuner while the first segment was fixed. In addition to the structural simulations, a bellow tuner prototype of niobium with one and a half segments was built and mechanically tested. Figure 7 shows the results from measurements at room temperature in comparison to the simulated values. Because of welding seams, which could not be considered in the simulations, the measured tuner deformation is approximately 3 times bigger.

At IAP Frankfurt and in cooperation with GSI and Helmholtz Institute Mainz (HIM), the design effort for a cw operated heavy ion linac has started. This dedicated linac will be applied to nuclear chemistry and to the production of super heavy elements especially. It has to provide ion beams with a mass-to-charge-ratio $(A / q)$ up to 6 and energies up to $7.3 \mathrm{AMeV}$. Above an energy of 3.5 AMeV the linac is energy variable. Because of the required cw operation the main linac will be superconducting. The front end will consist of the existing high charge injector (HLI) (108.48 MHz, 1.4 AMeV) which is presently being upgraded for the corresponding duty cycle. The main 

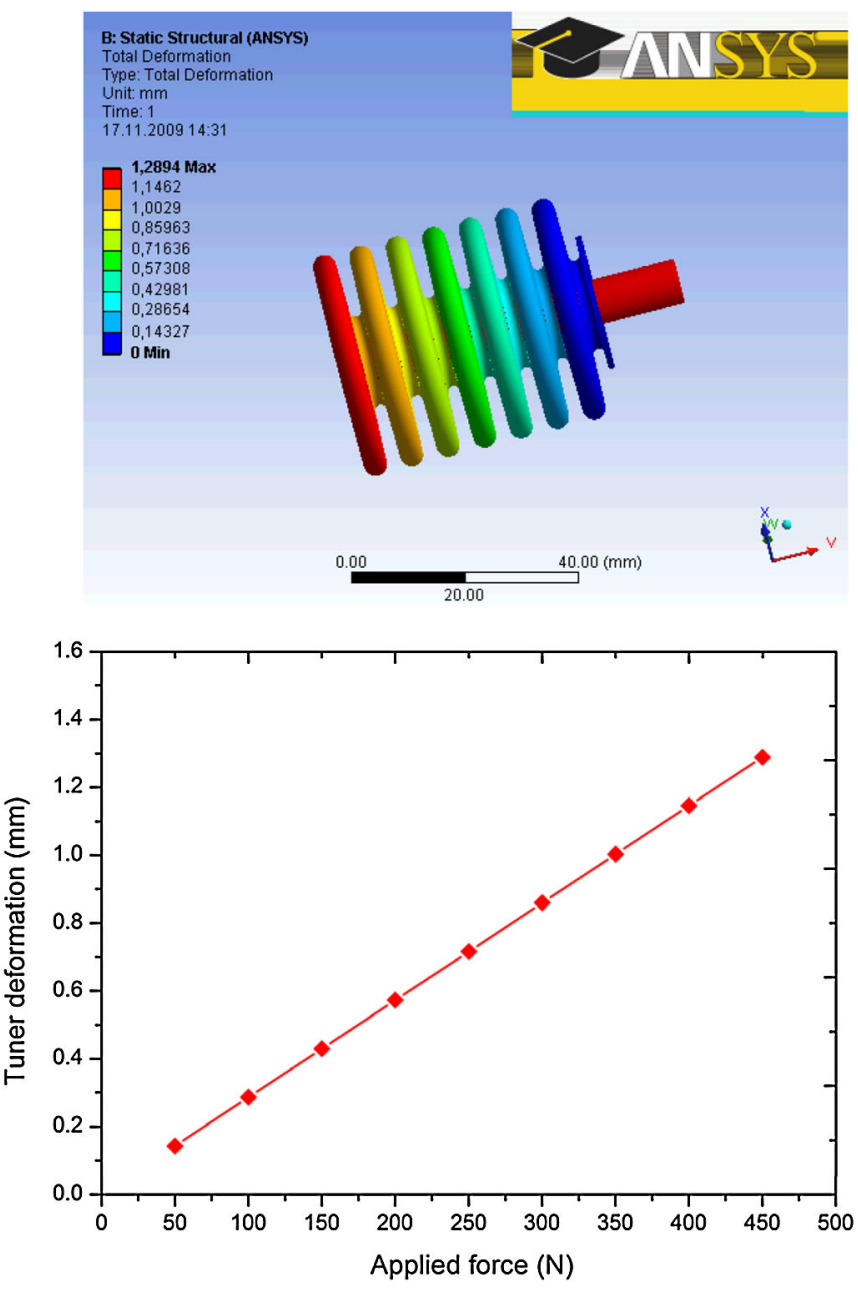

FIG. 6. (Color) Structural ANSYS simulation of a bellow tuner model (top) and deformation of the bellow tuner depending on several forces (bottom) [17].

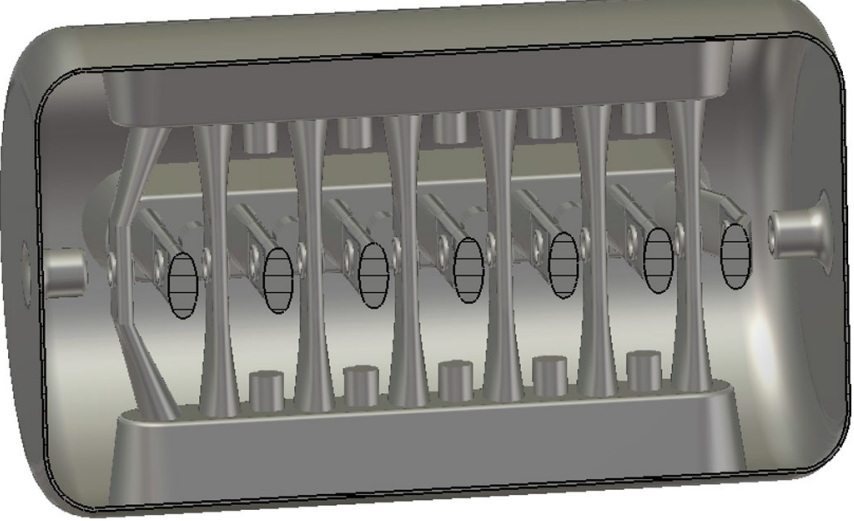

FIG. 8. Preliminary layout of the $217 \mathrm{MHz} \mathrm{CH}$ cavity for the $\mathrm{cw}$ heavy ion linac for an injection energy of $1.4 \mathrm{AMeV}$.

TABLE III. Parameters of the first superconducting CH cavity for the heavy ion linac.

\begin{tabular}{lcc}
\hline \hline$\beta$ & $\mathrm{MHz}$ & 0.059 \\
Frequency & $\mathrm{mm}$ & 217 \\
Total length & $\mathrm{mm}$ & 689 \\
Cavity diameter & & 413 \\
Accelerating cells & $\mathrm{MV} / \mathrm{m}$ & 15 \\
$E_{a}$ & & 5.1 \\
$E_{p} / E_{a}$ & 5.4 \\
$B_{p} / E_{a}$ & $\mathrm{mT} /(\mathrm{MV} / \mathrm{m})$ & 5.5 \\
$G$ & $\Omega$ & 52 \\
$R_{a} / Q_{0}$ & $\Omega$ & 3394 \\
$R_{a} R_{s}$ & $\mathrm{k} \Omega^{2}$ & 176 \\
\hline \hline
\end{tabular}
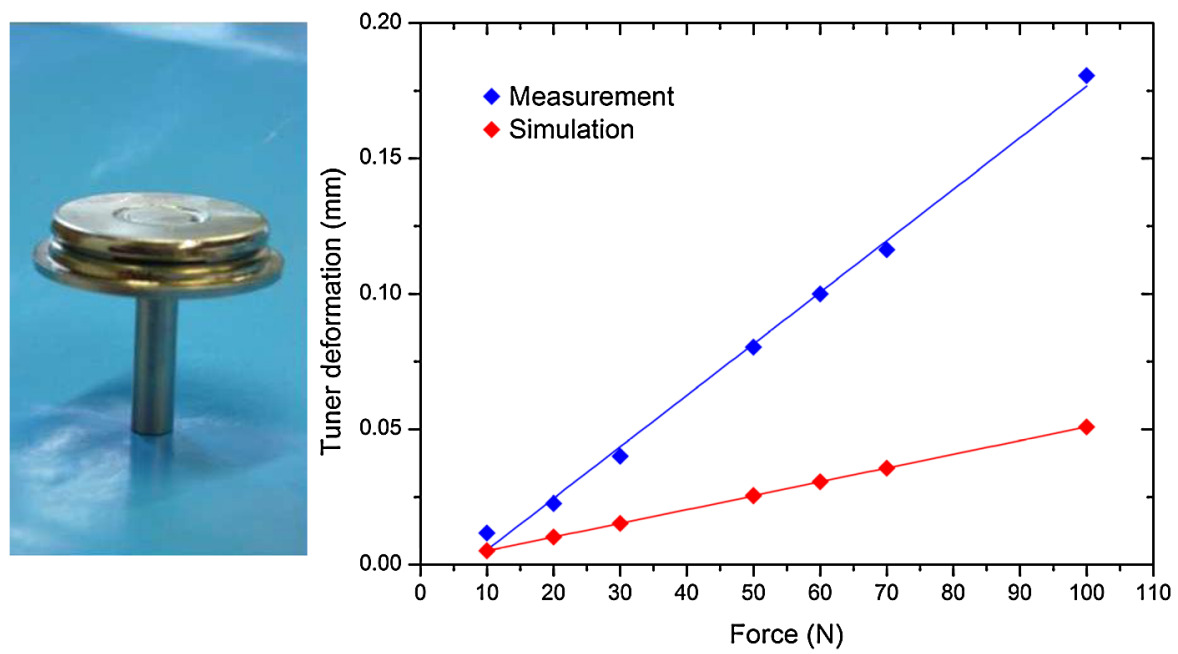

FIG. 7. (Color) Bellow tuner prototype of niobium with one and a half segments (left) and related results from measurements at room temperature in comparison to the simulations (right). 


\section{CW LINAC}

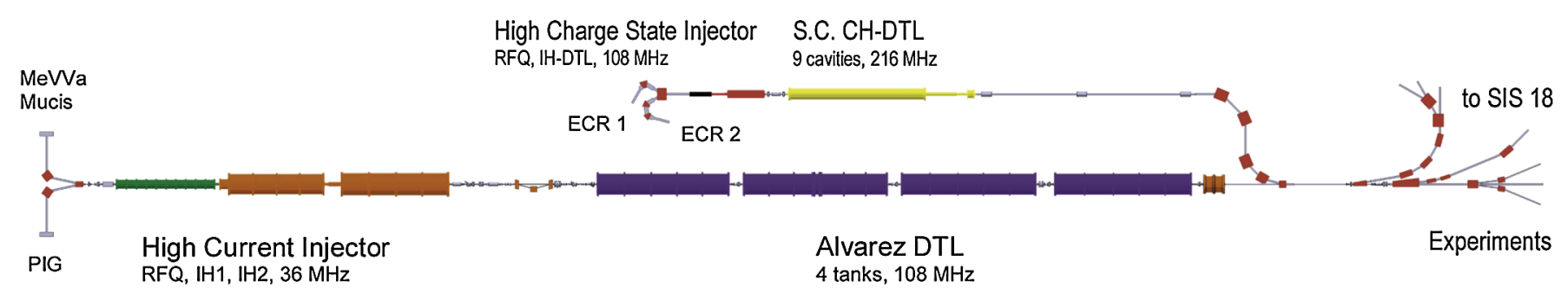

FIG. 9. (Color) Installation study of the designed $\mathrm{cw}$ linac in parallel to the GSI UNILAC.

acceleration of approximately $35 \mathrm{MV}$ will be done by a superconducting $\mathrm{CH}$ linac of nine cavities operated at $217 \mathrm{MHz}$.

The first superconducting $\mathrm{CH}$ cavity (shown in Fig. 8) of the main linac is currently under design and will be based on the EQUUS beam dynamics concept. It is planned to test the cavity with beam from the $1.4 \mathrm{AMeV}$ HLI. The geometrical properties are adapted to the $325 \mathrm{MHz} \mathrm{CH}$ cavity and first preliminary rf simulations could be achieved (see Table III). Figure 9 gives an overview of the $\mathrm{cw}$ heavy ion linac layout and its integration in parallel to the GSI UNILAC.

\section{THE EUROTRANS PROJECT}

The EUROTRANS project, supported by the European Union, is another high power application favoring superconducting $\mathrm{CH}$-cavity technology. It is proposed for the transmutation of high level nuclear waste using an accelerator driven system (ADS) with an efficient high-current cw linac [2]. One major issue of such an accelerator system is reliability and fault tolerance to reduce the number of unwanted beam trips. To avoid activation of the machine, beam losses have to be minimized. The EUROTRANS driver linac has to deliver a $600 \mathrm{MeV}$ proton beam with a beam current of $2.5 \mathrm{~mA}$, which can be increased up to $4 \mathrm{~mA}$ during the burning process of the fuel. The beam is transported to a spallation target consisting of liquid metal with a beam power of either 1.5 or $2.4 \mathrm{MW}$ depending on the beam current. Figure 10 shows the present reference design for the $600 \mathrm{MeV}$ EUROTRANS proton driver linac, proposed by IAP of Frankfurt University. A layout of the $17 \mathrm{MeV}$ front end, containing four superconducting $\mathrm{CH}$ cavities, is shown in Fig. 11.

In order to assure extremely high reliability of operation (less than 3-10 beam trips with $t>1 \mathrm{~s}$ per year), the EUROTRANS injector consists of two identical, redundantly designed, $17 \mathrm{MeV}, 352 \mathrm{MHz}$ front ends. Both front ends are in operation but only one injector delivers the beam to the main linac. In case of a beam trip in this injector, which is unfixable within a short time $(t<1 \mathrm{~s})$, the second injector will deliver the beam. Above $17 \mathrm{MeV}$ it is possible to keep the beam on target even in case of a cavity failure by the so-called dynamic rf compensation. In this context the voltage of the neighboring cavities is increased and the phase is adjusted to keep the beam parameters within its specifications [12]. Each front end consists of an electron cyclotron resonance ion source, a

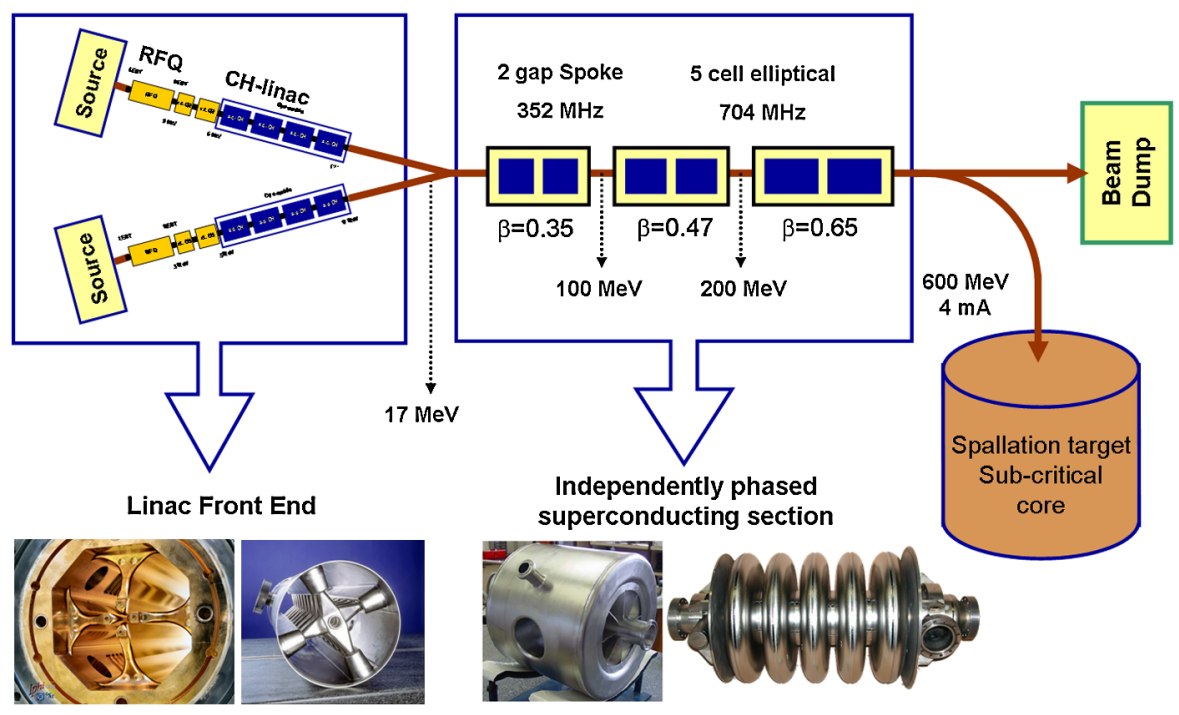

FIG. 10. (Color) Reference design of the $600 \mathrm{MeV}$ EUROTRANS proton driver linac. 


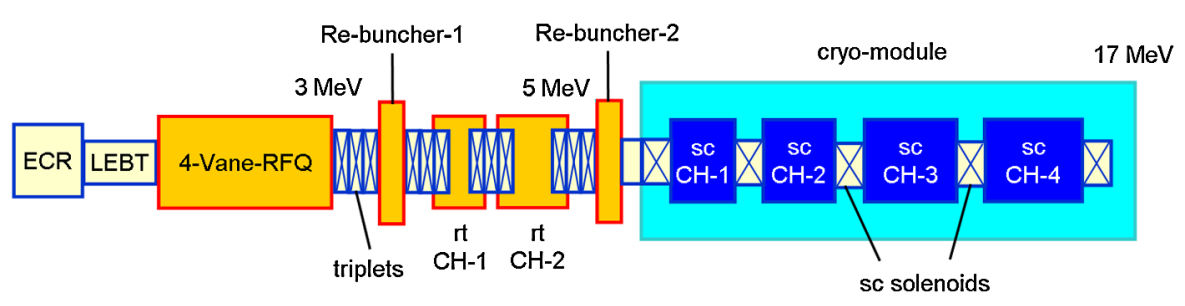

FIG. 11. (Color) Layout of the $17 \mathrm{MeV}$ EUROTRANS front end.

$3 \mathrm{MeV}$ 4-vane radio frequency quadrupole (RFQ), two room temperature $\mathrm{CH}$ cavities up to $5 \mathrm{MeV}$, and four superconducting CH-DTL (drift tube linac), which accelerate the beam up to $17 \mathrm{MeV}$. The room temperature cavities are foreseen to prepare the beam for the following superconducting $\mathrm{CH}$ structures. They are acting as beam loss filters and avoid a breakdown of superconductivity. The intermediate energy part from $17-100 \mathrm{MeV}$ consists of independently phased superconducting spoke cavities. Followed by two groups of superconducting elliptical five-cell cavities, the beam energy will be increased to $600 \mathrm{MeV}$.

\section{SUPERCONDUCTING CH DEVELOPMENT FOR THE EUROTRANS INJECTOR LINAC}

The main acceleration of the EUROTRANS front end will be provided by four superconducting $\mathrm{CH}$ cavities. In order to accomplish the requirements of this injector, the related superconducting cavities have been optimized accordingly $[13,14]$, based on the new design of the $325 \mathrm{MHz}$ cavity. A layout of the first superconducting EUROTRANS cavity is shown by Fig. 12.

Each cavity is equipped with a well designed velocity $(\beta)$ profile. Large power couplers up to $250 \mathrm{~kW}$ have been built in the cavities between the stems. Via integration of inclined stems, the end cell length and unwanted drift sections could be reduced significantly while reaching a flat field distribution on the beam axis as well as a high beam quality. A flat field distribution is necessary to reach an optimal acceleration and to reduce the peak fields. To avoid beam losses and activation of accelerator components, a high beam quality is essential. The minimization of unnecessary drift sections decreases the total cavity length by about $20 \%-25 \%$ without changing the voltage and peak fields [15].

Applying the same tuning concept as used for the $325 \mathrm{MHz}$ cavity described in Sec. I, the superconducting EUROTRANS CH cavities will contain seven static tuners, one fast and two slow bellow tuners. Figure 13 shows the simulated frequency range of the ten tuners for the first superconducting cavity. Based on this tuning range the working points for the static and the bellow tuners have been chosen with the aim to maximize the frequency gain while keeping the tuner height and the electrical peak fields to a minimum. For an adequate frequency adjustment during fabrication, a maximum height of $25 \mathrm{~mm}$ for the static tuners is foreseen. Table IV contains the calculated frequency shift and the total range for the fast and the slow bellow tuners at a working point of $35 \mathrm{~mm}$, which is sufficient for the cavity tuning during beam operation.

The electric field distribution on beam axis was optimized for every cavity by adjusting the gap-to-cell-length ratio $(g / l)$. Figure 14 shows the simulated field distribution

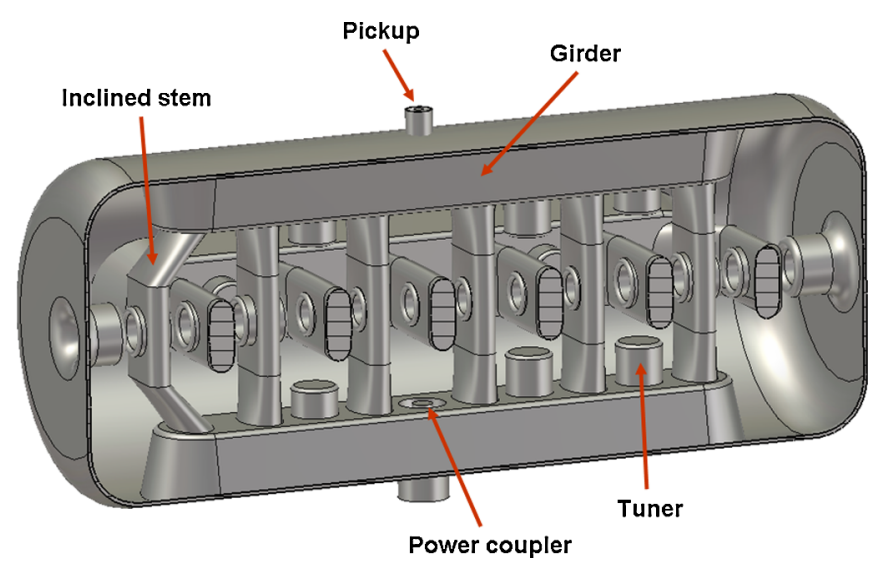

FIG. 12. (Color) Layout of the first superconducting EUROTRANS CH cavity (simulation with MICROWAVE STUDIO [18]).

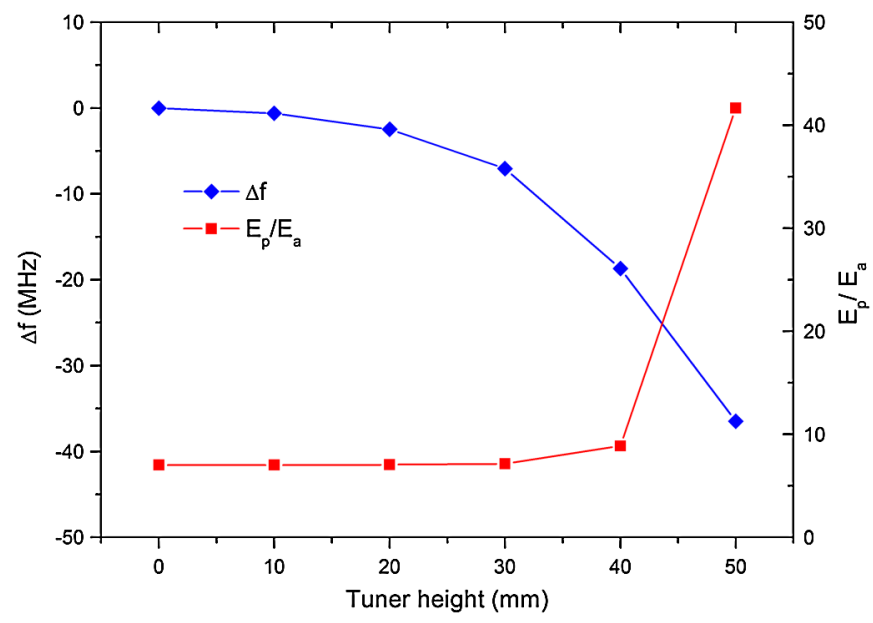

FIG. 13. (Color) Simulated tuning range of ten hypothetic tuners operated in parallel and electrical peak fields appearing at the tuner surface. 
TABLE IV. Frequency shift and total range of the bellow tuners for the first cavity.

\begin{tabular}{lcc}
\hline \hline Parameter & Slow tuner & Fast tuner \\
\hline No. & 2 & 1 \\
Frequency shift & $114 \mathrm{kHz} / \mathrm{mm}$ & $112 \mathrm{~Hz} / \mu \mathrm{m}$ \\
Total range & $\pm 228 \mathrm{kHz}( \pm 1 \mathrm{~mm})$ & $\pm 560 \mathrm{~Hz}( \pm 5 \mu \mathrm{m})$ \\
\hline \hline
\end{tabular}

before and after the optimization (above) and the related gap with the corresponding drift tube length for the flat field of the first superconducting EUROTRANS CH cavity (bottom). The length of tube number three results from the integrated rebuncher section of the KONUS dynamics.

After passing the last room temperature $\mathrm{CH}$ cavity the first superconducting $\mathrm{CH}$ structure will increase the beam energy up to $7.5 \mathrm{MeV}$ provided by an effective voltage of $2.5 \mathrm{MV}$. At a design $Q$ value of $2 \times 10^{8}$, a power of $18 \mathrm{~W}$ is

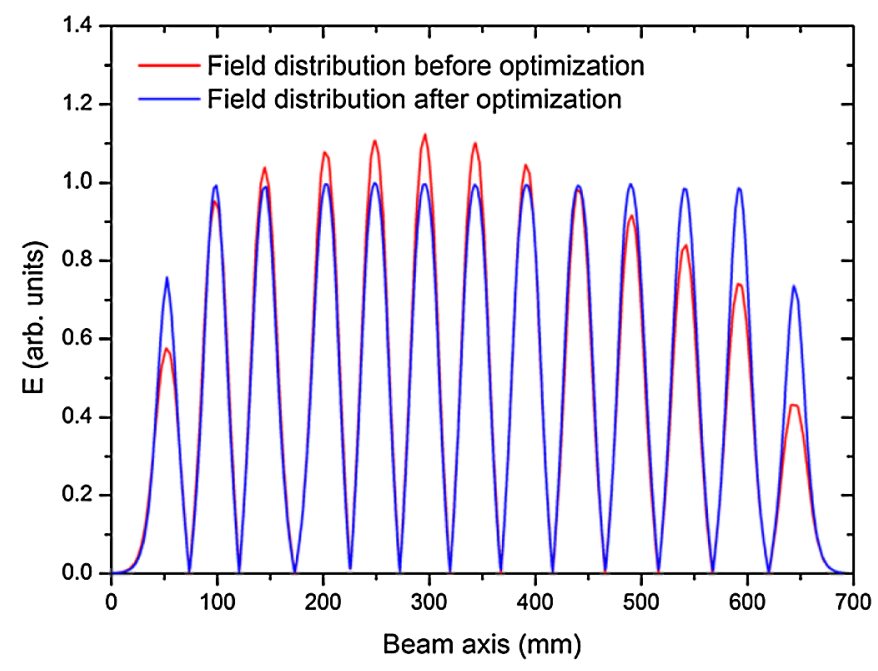

Tube No.

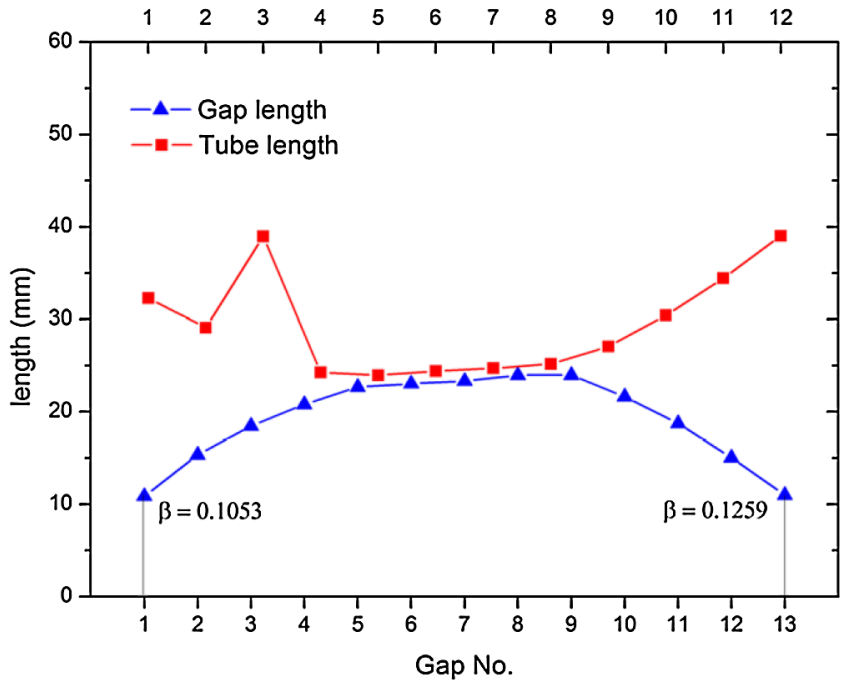

FIG. 14. (Color) Simulation of the electric field distribution before and after the optimization (above), related gap and drift tube length for the flat field (bottom).

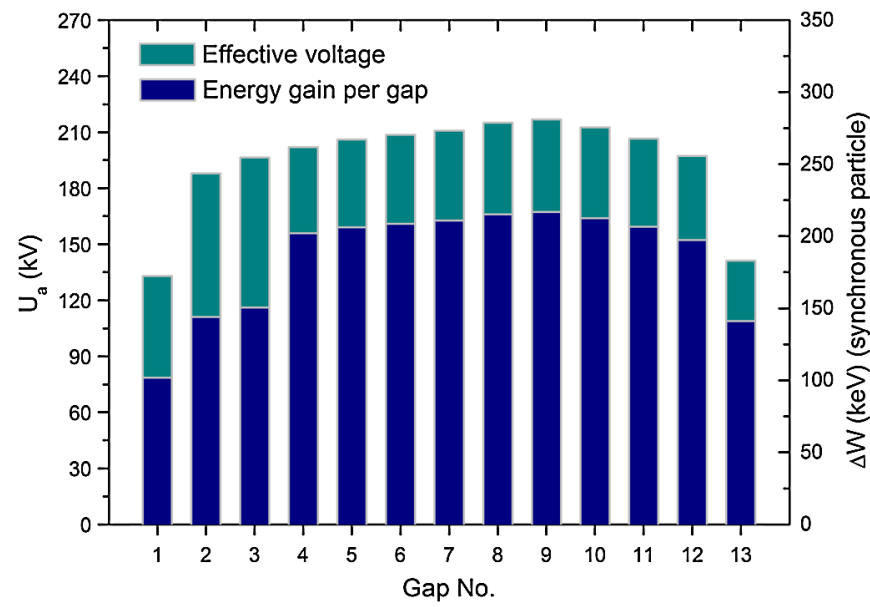

FIG. 15. (Color) Effective voltage on beam axis and related energy gain per gap for a synchronous particle of the first $\mathrm{CH}$ cavity.

required without beam. Because of the optimized electric field, an effective voltage distribution on the beam axis has been achieved, which performs the required energy gain per gap (see Fig. 15). A technical drawing of the first $\mathrm{CH}$ structure optimized for the EUROTRANS front end is shown by Fig. 16 while Table V summarizes the current main parameters for all superconducting $\mathrm{CH}$ cavities.

Based on the optimized superconducting as well as the room temperature $\mathrm{CH}$ cavities, beam dynamic simulations have been performed for the DTL part of the EUROTRANS injector (not including the RFQ part) [16]. The transverse beam envelopes at $5 \mathrm{~mA}$ from the preliminary design are shown in Fig. 17, from which one can see that a large safety margin for the beam transport is available throughout the injector linac. Figure 18 plots the transverse particle distributions at the two ends of the

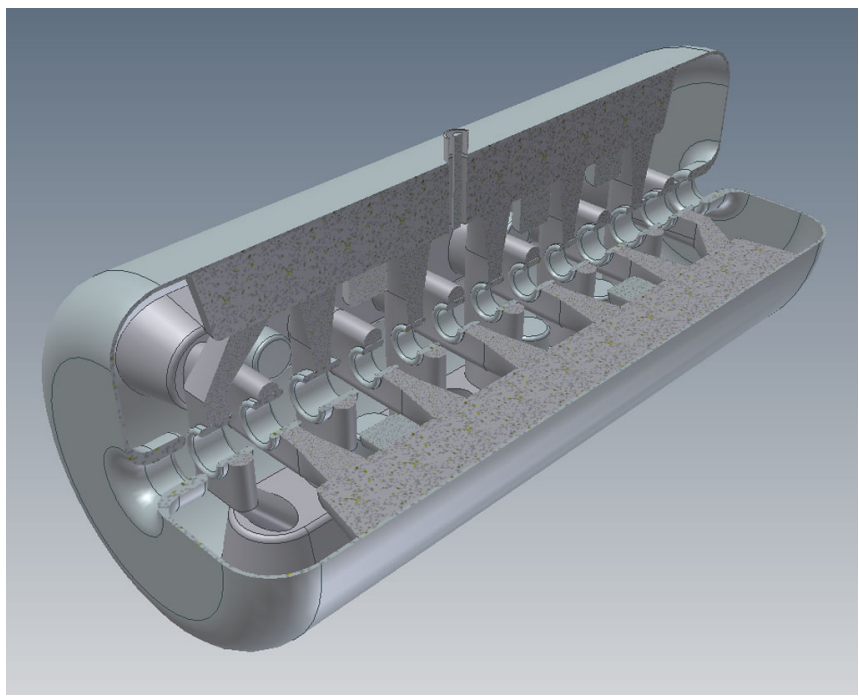

FIG. 16. First superconducting $\mathrm{CH}$ cavity optimized for the EUROTRANS front end. 
TABLE V. Main parameters of the superconducting $\mathrm{CH}$ cavities for the EUROTRANS front end.

\begin{tabular}{lccccc}
\hline \hline Parameter & Unit & $\mathrm{CH}-1$ & $\mathrm{CH}-2$ & $\mathrm{CH}-3$ & $\mathrm{CH}-4$ \\
\hline $\bar{\beta}$ (mean value) & & 0.117 & 0.138 & 0.159 & 0.178 \\
Frequency & $\mathrm{MHz}$ & 352 & 352 & 352 & 352 \\
Energy range & $\mathrm{MeV}$ & $5-7.4$ & $7.4-10.4$ & $10.4-13.9$ & $13.9-17.4$ \\
Total length & $\mathrm{mm}$ & 696.6 & 862.9 & 1014.0 & 1124.3 \\
Cavity diameter & $\mathrm{mm}$ & 286.1 & 300.8 & 316.3 & 332.5 \\
Aperture diameter & $\mathrm{mm}$ & $25-30$ & 30 & 40 & 40 \\
Accelerating cells & & 13 & 14 & 14 & 14 \\
Bellow tuner & & 3 & 3 & 3 & 3 \\
Fixed tuner & & 7 & 7 & 7 & 7 \\
$G$ & $\Omega$ & 56 & 58 & 60 & 63 \\
$R_{a} / Q_{0}$ & $\Omega$ & 1775 & 2143 & 1831 & 1999 \\
$R_{a} R_{s}$ & $\mathrm{k} \Omega^{2}$ & 99 & 124 & 110 & 125 \\
$Q_{0}(\mathrm{BCS})$ & & $1.4 \times 10^{9}$ & $1.5 \times 10^{9}$ & $1.5 \times 10^{9}$ & $1.6 \times 10^{9}$ \\
$Q_{0}$ (goal) & & $2 \times 10^{8}$ & $2 \times 10^{8}$ & $2 \times 10^{8}$ & $2 \times 10^{8}$ \\
$E_{a}(\beta \lambda$ definition) & $\mathrm{MV} / \mathrm{m}$ & 3.9 & 3.9 & 3.9 & 3.6 \\
$E_{p}$ & $\mathrm{MV} / \mathrm{m}$ & 26.5 & 28.1 & 28.5 & 27.4 \\
$E_{p} / E_{a}$ & & 6.8 & 7.2 & 7.3 & 7.6 \\
$B_{p}$ & $\mathrm{mT}$ & 39.8 & 35.9 & 45.2 & 36 \\
$B_{p} / E_{a}$ & $\mathrm{mT} /(\mathrm{MV} / \mathrm{m})$ & 10.2 & 9.2 & 11.6 & 10 \\
$U_{a}\left(\right.$ for goal $\left.Q_{0}\right)$ & $\mathrm{MV}$ & 2.5 & 3.2 & 3.7 & 3.8 \\
$P_{c}$ (for & 18 & 24 & 37 & 36 \\
\hline \hline
\end{tabular}
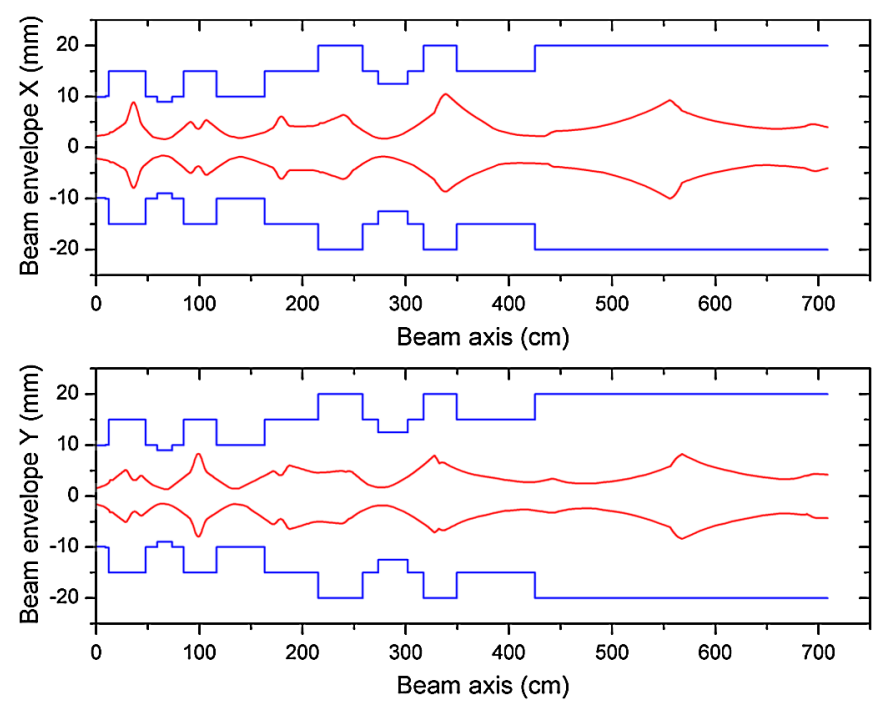

FIG. 17. (Color) Transverse beam envelopes for the DTL part of the EUROTRANS injector.

DTL; the corresponding $100 \%$ rms emittance growths are about $15 \%$.

\section{SUMMARY AND OUTLOOK}

The superconducting $\mathrm{CH}$ structure is a multicell drift tube cavity for the low and medium energy range. It is an excellent candidate for the efficient acceleration in high power proton and ion applications because the large energy gain per cavity leads to high real estate gradients and to a significant reduction of required accelerator components.
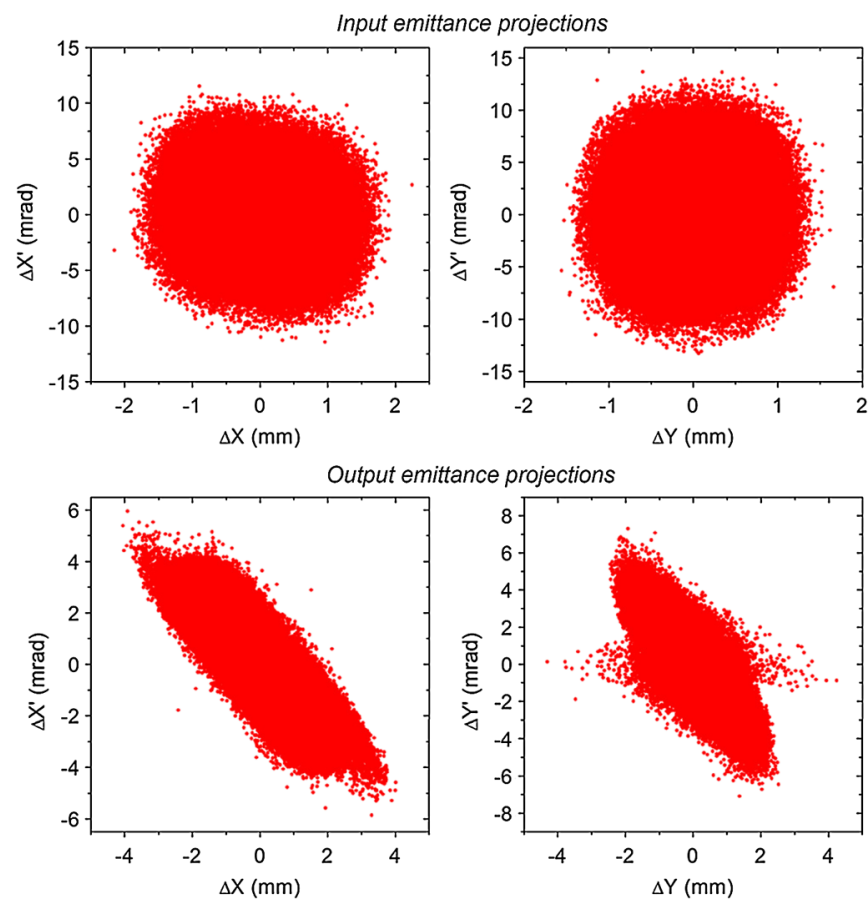

FIG. 18. (Color) Transverse emittance projections at the input and output of the EUROTRANS DTL part for $5 \mathrm{~mA}$.

A first prototype cavity has been built and tested successfully. At present, the construction of a $325 \mathrm{MHz} \mathrm{CH}$ cavity is in progress while another $217 \mathrm{MHz}$ cavity is under development. Both cavities will be tested with beam at GSI.

The reference design of the $352 \mathrm{MHz}, 600 \mathrm{MeV}$ EUROTRANS proton driver linac using four superconducting $\mathrm{CH}$ cavities for the $17 \mathrm{MeV}$ front end was proposed by IAP, Frankfurt University. In this context the superconducting $\mathrm{CH}$ cavities were designed and optimized accordingly. Both room temperature $\mathrm{CH}$ cavities have already been designed. Further simulations have to be done to improve the longitudinal beam dynamics in an iterative process. It is planned to build the first room temperature EUROTRANS CH cavity and test it with full rf power in the next years.

\section{ACKNOWLEDGMENTS}

This work has been supported by BMBF Contracts No. 06F134I and No. 06FY9089I, EU EUROTRANS Contract No. 516520-FI6W, EU-CARE-HIPPI No. RII3CT-2003-506395, and by the Hessian Research Initiative LOEWE-HIC for FAIR. The authors would like to thank the company RI, Bergisch Gladbach, Germany, for the excellent work on technical drawings as well as in the fabrication of the prototype cavities. In addition, they would like to thank the technical staff of the IAP in Frankfurt, especially D. Bänsch, I. Müller, and S. Reploeg. 
[1] U. Ratzinger et al., Nucl. Instrum. Methods Phys. Res., Sect. A 415, 229 (1998).

[2] The European Technical Working Group on ADS, A European Roadmap for Developing Accelerator Driven Systems (ADS) for Nuclear Waste Incineration (ENEA, Rome, Italy 2001).

[3] H. Podlech et al., in Proceedings of the HPPA5 Workshop, Mol, Belgium 2007, pp. 115-124.

[4] U. Ratzinger, in Proceedings of the 1991 Particle Accelerator Conference, 1991, pp. 567-571.

[5] T. P. Wangler, RF Linear Accelerators (Wiley, New York, 2008).

[6] Minaev et al., Phys. Rev. ST Accel. Beams 12, 120101 (2009).

[7] H. Podlech et al., Phys. Rev. ST Accel. Beams 10, 080101 (2007).

[8] H. Podlech, Habilitationsschrift, University of Frankfurt, Germany, 2009.

[9] A. Bechtold et al., in Proceedings of the 2007 Superconducting RF Workshop, Bejing, China, p. 618.

[10] H. Podlech et al., in Proceedings of the 2009 Super- conducting RF Workshop, Berlin, Germany, pp. 473-478.

[11] M. Busch et al., in Proceedings of the 2009 Particle Accelerator Conference, Vancouver, Canada, 2009 (unpublished).

[12] J.-L. Biarotte et al., Phys. Rev. ST Accel. Beams 11, 072803 (2008).

[13] F. Dziuba et al., in Proceedings of the International Topical Meeting on Nuclear Research Applications and Utilization of Accelerators, 2009, Vienna (IAEA, Vienna, 2010), ISBN 978-92-0-150t.410-4.

[14] F. Dziuba et al., in Proceedings of the 2009 Superconducting RF Workshop, Berlin, Germany (Ref. [10]), pp. 578-581.

[15] H. Podlech et al., in Proceedings of the 11th European Particle Accelerator Conference, Genoa, 2008 (EPS-AG, Genoa, Italy, 2008), pp. 901-903.

[16] C. Zhang (unpublished).

[17] ANSYS Multiphysics, http://www.ansys.com.

[18] CST MICROWAVE STUDIO, http://www.cst.com. 